\title{
In the dark II: Spatial choice when access to extrinsic spatial cues is eliminated
}

\author{
MICHAEL F. BROWN and JONATHAN A. MOORE \\ Villanova University, Villanova, Pennsylvania
}

\begin{abstract}
Rats were tested in a specially constructed radial-arm maze that eliminated access to extramaze visual cues and allowed any effects of intramaze cues to be controlled. Despite this, choice accuracy was controlled by the spatial location of previously visited arms. Part of this control was attributed to vestibular or kinesthetic cues. This conclusion was corroborated by the finding that when explicit visual cues were moved from their standard (trained) spatial locations to novel locations, control of spatial choices was completely disrupted. The latter finding indicates that cues intrinsic to the rat (kinesthetic or vestibular information) and cues extrinsic to the rat (visual stimuli) operate in an integrated fashion.
\end{abstract}

In the radial-arm maze (RAM; Olton \& Samuelson, 1976), rats accurately discriminate between locations that they have previously visited and locations they have not previously visited within an experimental trial. This is shown by the fact that they avoid revisits to maze arms, thereby increasing the efficiency with which they deplete the small bits of food placed at the end of each maze arm prior to each trial.

The results of numerous experiments have shown that, in a standard RAM apparatus, this ability is under stimulus control of the extra-apparatus visual cues provided by objects in the laboratory room containing the RAM. For example, Zoladek and Roberts (1978) showed that blinded rats performed at levels of choice accuracy in the RAM that were substantially lower than those of nonblinded rats. Mazmanian and Roberts (1983) showed that choice accuracy in the RAM is determined by the extent to which the extramaze environment is visible from the threshold leading to each arm. Perhaps the clearest and most influential set of results indicating the control of choice in the RAM by visual cues is that of Suzuki, Augerinos, and Black (1980). They used a maze in which the extra-apparatus cues could be precisely controlled: The maze was surrounded by a large circular curtain and the only extramaze cues were those explicitly provided. Two findings provide strong evidence that extramaze visual cues are critical for RAM performance. First, if the cues were moved (rotated) during a short trial interruption, the choice behavior of the rats "followed the cues" - that is, the rats chose the arms corresponding to cues that had not received a visit prior to trial interruption. Second, if the spatial relations among the cues were disrupted (by haphazardly rearranging the cues in relation to one an-

Experiment 3 was conducted in partial fulfillment of the requirements for J.A.M.'s master's degree under the supervision of M.F.B. Correspondence should be addressed to M. F. Brown, Department of Psychology, Villanova University, Villanova, PA 19085 (e-mail: mbrown@email.vill.edu). other), choice accuracy was substantially reduced. The latter fact has been widely interpreted as indicating that cues are not represented independently, but rather are represented in the context of a "cognitive map" of the extraapparatus environment (but see Brown, 1992, p. 56).

Although it seems clear that visual cues are critical for the high levels of choice accuracy found in the standard RAM, it also appears that visual cues are not necessary for above-chance levels of choice accuracy. Zoladek and Roberts (1978) noted that their blinded rats performed at above-chance levels of choice accuracy and suggested that "blind rats may have been making use of internal vestibular and/or kinesthetic cues to keep track of positions in space" (p. 81). Others have also reported that although blinded rats or rats tested in complete darkness perform less accurately than intact rats, they appear to avoid revisits to maze arms to some extent (Dale \& Innis, 1986; Foreman, 1985; Goodale \& Dale, 1981; Whishaw \& Tomie, 1989). Etienne, Sitbon, Dahn-Hurni, and Maurer (1994) also reported that hamsters tested under conditions of total darkness performed in a RAM at above-chance levels, although their choices were less accurate than those of hamsters tested in an illuminated environment.

The latter results are intriguing because they suggestand have been interpreted as showing (Etienne et al., 1994; Zoladek \& Roberts, 1978)-that rats and hamsters can determine their present and past locations in the RAM using intrinsic vestibular or kinesthetic information. The hypothetical process of "path integration" or "dead reckoning" (Etienne, 1992; Gallistel, 1990) involves the use of such vestibular or kinesthetic information about the distance and direction traveled to determine the relation between the current location and a location from which one has traveled. There is a great deal of evidence that a wide range of animals use dead reckoning to return to a reference location following an episode of travel (see Gallistel, 1990, and Potegal, 1982, for reviews). However, to propose that animals use dead reckoning to solve the RAM is to propose a much more com- 
plex process. In the RAM, multiple locations that have been previously visited must be discriminated from multiple locations that have not yet been visited. Thus, the hypothetical dead reckoning process involved in RAM performance would have to code multiple locations during a long and complex movement path. Those locations would then have to be discriminated from within the central arena of the maze (i.e., from a location other than that where the food was discovered).

With the exception of Zoladek and Roberts's (1978) data, the data from experiments in which rats have been tested in the RAM in the absence of visual cues are open to interpretations other than dead reckoning. Two alternatives to dead reckoning are generally available. The first is that rats leave a physical trace behind as they visit arms (most likely an odor trail), and this trail is used as a cue to discriminate visited and unvisited arms. In the standard RAM, this explanation for above-chance levels of choice accuracy has been ruled out in a number of ways, most often by rotating the maze during an interruption in each trial (see, e.g., Olton \& Collison, 1979). Although it seems clear that such physical traces cannot explain performance in the standard RAM, it remains possible that elimination of visual cues might elicit the use of such cues. The second alternative to dead reckoning as an explanation of choice accuracy when visual cues are eliminated is the use of a consistent pattern of movement from arm to arm in such a way that the probability of revisits is reduced. For example, a rat might consistently move to the adjacent arm on its right as it leaves each maze arm. Such a behavioral tendency would reduce errors without requiring discrimination between visited and unvisited maze arms. Again, this explanation has been consistently ruled out in the standard RAM, but might be involved in the atypical experimental conditions under consideration here. In fact, a strong adjacent arm tendency in blinded rats was found by Dale and Innis (1986). A standard technique for eliminating the role of such movement tendencies is to use a forced-choice procedure in which the rat first visits a randomly chosen set of maze arms and then freely chooses among all arms of the maze. It is important in such experiments that the behavioral measure used isolate the ability of the rat to discriminate between the arms to which it was forced and those to which it was not forced.

The experimental procedure of Zoladek and Roberts (1978) included both a forced-choice procedure and midtrial maze rotation. However, later experiments involving deprivation of visual cues have failed to include a forced-choice procedure (Dale \& Innis, 1986), midtrial maze rotation (Etienne et al., 1994), or both (Foreman, 1985; Goodale \& Dale, 1981; Whishaw \& Tomie, 1989), thereby allowing the possibility that physical traces, response patterns, or both might explain above-chance levels of choice accuracy.

Experiments from our laboratory (Brown, 1992, 1993; Brown, Rish, VonCulin, \& Edberg, 1993) have examined the choice-related behavior of rats in the central arena of
RAMs, particularly behaviors related to the acquisition of visual information corresponding to maze arms (i.e., observing responses). In the standard RAM, the choice process can be explained as a sequence of yes/no decisions, the target of each being an individual maze arm (Brown, 1992). There is no evidence that the target of these yes/no decisions is more likely to be a correct maze arm than would be expected on the basis of chance. In other words, rats move about in the central arena without being controlled by the location of baited arms. It is only after they examine the visual stimulus corresponding to an arm that the stimulus controls their spatial choice behavior. On the other hand, when access to the visual cues in the extra-apparatus environment is restricted (Brown et al., 1993), the movement of rats in the central arena of the maze is guided toward the location of correct arms. In other words, rats navigate toward the location of goals in the absence of spatial cues when access to such cues is restricted.

Such spatial guidance of the rat from one maze arm to the next can be explained in at least two ways. First, cues available in the central arena of the maze (odor or visual cues that differentiate maze arms) might provide an extrinsic cue that directs movement toward maze arms. On the basis of experiments in which the RAM was rotated, Brown et al. (1993) argued that such intramaze cues can explain spatial guidance under some experimental conditions, but that some other process is also required to explain their results. They emphasized that this other process must include a representation of the spatial relations among the maze arms (i.e., a cognitive map). However, this is just part of the cognitive machinery necessary to get the rat from one part of the central arena to another, without the benefit of visual cues. In addition to a representation of where the maze arms are in relation to one another (an allocentric representation), there must be a representation of where the rat is in relation to the maze (an egocentric representation). It is this part of the process that may correspond to dead reckoning. In a maze with restricted access to extramaze cues, the rat could keep track of its current position in the central arena using intrinsic (kinesthetic or vestibular) cues.

Brown and Bing (1997) used a RAM in which the central arena and part of each maze arm were completely enclosed. As in the experiments of Brown et al. (1993), extra-apparatus visual cues were not available until the rat had pushed open a hinged door at the end of the enclosed portion of a maze arm. Brown and Bing's rats demonstrated very accurate levels of spatial choice when they were allowed to choose freely from among the 12 arms until all 12 had been chosen. However, when the rats were first forced to a randomly chosen set of 6 arms (the maze was then rotated, and the rat was allowed to choose freely from among the 12 arms-i.e., the forcedchoice procedure), the ability of rats to avoid revisits to the forced-choice spatial locations was no better than that expected by chance. Although this finding suggests that 


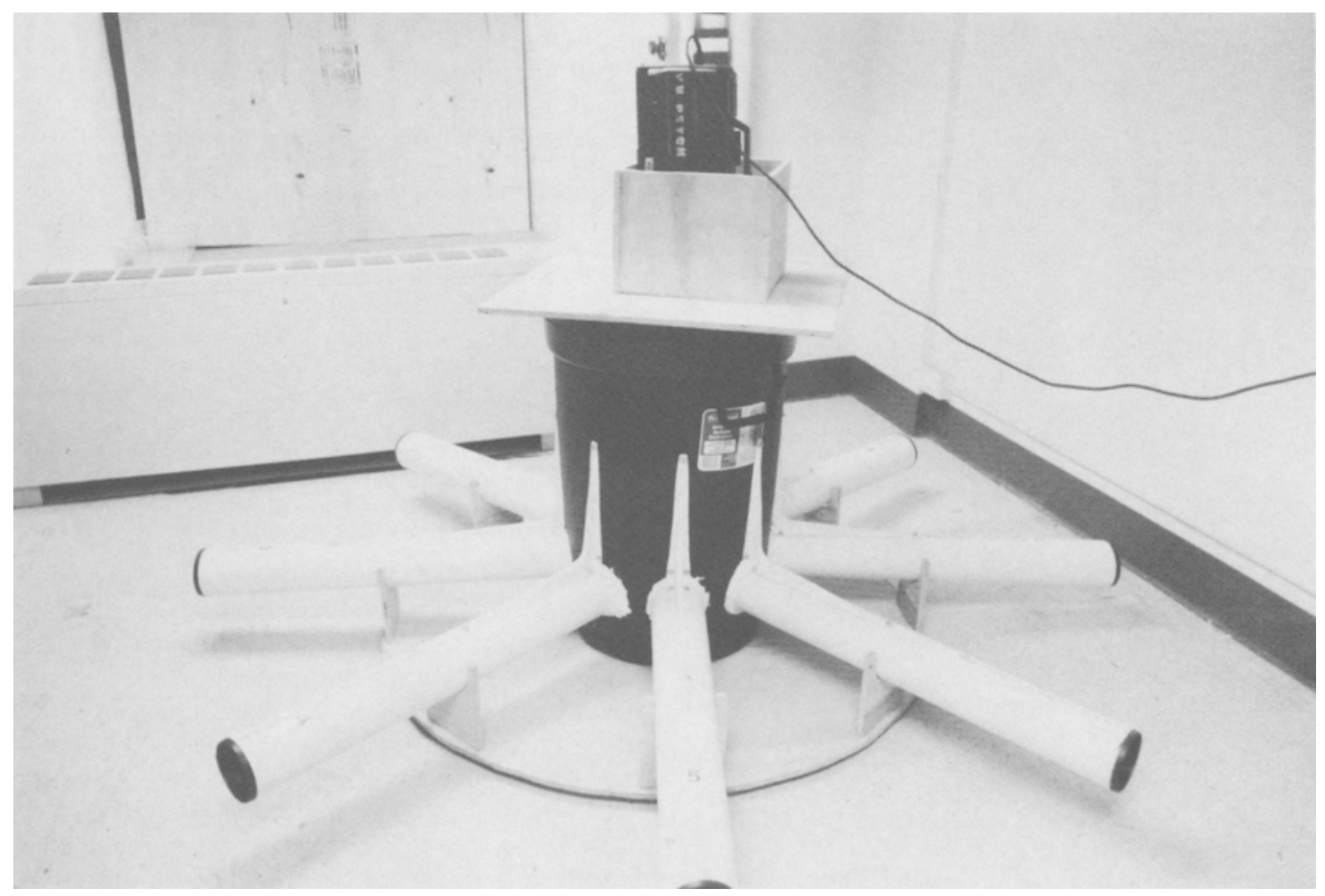

Figure 1. Photograph of the 8-arm maze used in the experiments.

intramaze cues (e.g., an odor trail) were controlling choices, an analysis of the source of errors showed clearly that this was not the case. Brown and Bing concluded that their rats were controlled by a complex amalgamation of intra- and extra-apparatus cues that was disrupted by rotation of the maze.

In the experiments of Brown et al. (1993) and Brown and Bing (1997), rats did not have access to extramaze cues from the central arena of the maze, but could gain access to those cues by opening doors leading to each maze arm, which they necessarily did before traveling to the end of a maze arm. Thus, extramaze visual cues and cues available in the central arena might both be used in such circumstances, and in fact this is what appears to have happened in these experiments. Brown et al. and Brown and Bing developed analytic techniques for examining the relative control of spatial location and intramaze cues. It would be of value to apply these analytic techniques to an experimental situation in which access to visual cues in a RAM is eliminated. This was the purpose of the present experiments. The procedures and analytic techniques used are similar to those used in the earlier experiments from our laboratory. However, the apparatus was one in which access to extramaze visual cues was eliminated both in the central arena and on the arms of the maze. Thus, any ability of the rat to avoid revisits to locations must be explained either in terms of extrinsic cues available inside the maze (e.g., odor or imperfections in the apparatus) or in terms of intrinsic cues (kinesthetic or vestibular information).

It is important to emphasize that our distinction between intrinsic cues (from inside the rat) and extrinsic cues (from outside the rat) is different from the distinction between extramaze cues and intramaze cues, which has been used in many studies using the RAM, including the most recent study from our laboratory (Brown \& Bing, 1997). Both intramaze and extramaze cues originate from outside the rat (and are therefore referred to as "extrinsic" cues). The present experiments were designed to distinguish the effects of intrinsic cues from both of these forms of extrinsic cues.

\section{EXPERIMENT 1}

The first experiment provided baseline information about the performance of rats in a specially designed RAM in which access to extramaze visual cues was eliminated. The maze arms were constructed using sections of translucent white polyvinylchloride (PVC) tubing, and the central arena was constructed using an opaque enclosed cylinder (a plastic refuse container). Light entered the apparatus through the PVC tubing, but was highly diffused by this material; thus any extramaze visual cues were eliminated. 


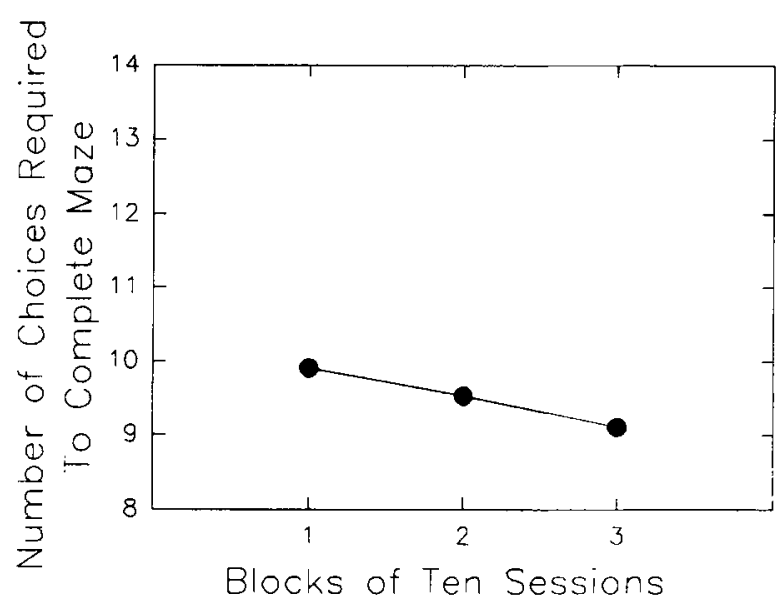

Figure 2. Choice accuracy during Experiment 1.

\section{Method}

Subjects. The subjects were 14 male Sprague-Dawley rats obtained from Harlan Sprague Dawley, Inc. (Indianapolis, IN). They were approximately 4 months of age and experimentally naive when the experiment began. The rats were housed in groups of 3 or 4 in a colony room with a $12: 12-h$ light:dark cycle. They began a restricted diet of $13 \mathrm{~g}$ per day of Purina Rat Chow 10 days prior to being placed in the apparatus.

Apparatus. The apparatus was the 8 -arm radial maze shown in Figure 1. The central arena was an opaque refuse container (Roughneck, Rubbermaid, Inc.), which was $77 \mathrm{~cm}$ tall and $44 \mathrm{~cm}$ in diameter at the end used as the floor of the maze. Eight holes were evenly spaced $(16 \mathrm{~cm}$ apart center-to-center) and centered $15 \mathrm{~cm}$ from the floor of the central arena. The floor of the central arena was formed by a $3-\mathrm{cm}$ layer of bedding material. Eight $78-\mathrm{cm}$ sections of white PVC tubing (10-cm interior diameter) were inserted into these holes, with the joints well caulked to ensure a light seal. The tubes were supported by braces and attached to a wooden turntable (mounted on a "lazy Susan"), allowing the entire apparatus to be rotated easily relative to the room. An end cap for each maze arm consisted of an opaque plastic flower pot, which was inserted into the maze arm so that its bottom extended $9 \mathrm{~cm}$ into the arm and served as the end of the arm from the rat's perspective. Small black plastic containers $(2.0 \mathrm{~cm}$ square and $1.5 \mathrm{~cm}$ deep, open at the top) were attached to the inside of these end caps and served as food cups. The end caps were easily removed to allow baiting (or unbaiting) the food cup. A lip around the top edge of the flower pots used as end caps provided a snug seal between the end cap and the maze arm. A plywood sheet served as a removable lid for the apparatus. A hole in the center of this lid allowed the lens of a camcorder (mounted on the lid) to protrude into the maze. The camcorder's view was displayed on a monitor, thereby allowing the choices of the rats to be observed as silhouettes of a rat moving into the translucent white circular areas of the display corresponding to each maze arm. Two windows in the room were covered with opaque material to ensure that the only light in the room was from a bank of four fluorescent tubes mounted on the ceiling directly above the maze.

Procedure. During each of 3 days prior to first placement in the maze, each rat was given $10-20$ of the $45-\mathrm{mg}$ sucrose pellets (Bio Serv, Inc., Frenchtown, NJ) to be used in the experiment. Three daily maze-exposure trials followed. During each of these trials, the maze was in a different orientation relative to the room. Sucrose pellets were placed in each food cup, scattered along the length of each maze arm, and (during the first two trials only) scattered around the perimeter of the central arena. Rats were placed in the center of the maze and allowed to explore the maze and consume pellets in groups of 3 or 4 .

Rats were then individually trained to obtain pellets from the ends of the maze arms. Prior to each trial, the maze was rotated into one of eight equally spaced orientations, randomly chosen. Two pellets were placed in each food cup. For some rats during some trials, 4-5 pellets were also placed on the bottom surface of the maze arm. The rat was first placed in the central arena by opening the lid, placing the rat in the center of the central arena, and replacing the lid as quickly as possible. Rats were placed in the maze in a consistent spatial orientation. Each rat was then allowed to choose from among the maze arms until all eight arms had been chosen, 24 choices had been made, or 13 min had elapsed. Daily training trials continued until each rat had consumed pellets from all food cups during a single trial. Daily test trials followed successful training and were identical to the training trials just described except that the maze was baited with only two pellets in each of the food cups. Thirty trials were conducted for each rat.

\section{Results}

A mean of 1.9 individual training trials were required for the rats to take pellets from the ends of maze arms (range $=1-5$ ). During the test trials, rats completed each trial by visiting all eight maze arms, with the exception of 1 rat that failed to complete two trials and 3 additional rats that failed to complete one trial each. Overall choice accuracy is shown in Figure 2 as the mean number of choices required to visit all eight maze arms (not counting the five trials just described) during each of three blocks of 10 trials each. Rats required substantially fewer choices to complete the maze than would be expected on the basis of chance (15-21, depending on the assumptions that one makes; see, e.g., Eckerman, 1980). The number of choices required to complete the maze declined over the course of testing $[F(2,26)=5.2, p<.05]$.

Table 1 shows, for each subject, the distribution of spatial separations between successive choices during the last block of 10 trials. Cases in which a chosen maze arm was spatially adjacent to that of the previous choice were assigned a value of 1 ; cases in which a chosen arm was separated from that of the previous choice by one

Table 1

Distribution of Spatial Separations Between Successive Choices During Last Block of 10 Trials in Experiment 1

\begin{tabular}{crccc}
\hline & \multicolumn{3}{c}{ Spatial Separation (Percentage of Choices) } \\
\cline { 2 - 5 } Rat & 1 Arm & 2 Arms & 3 Arms & 4 Arms \\
\hline 1 & 19.7 & 48.5 & 19.7 & 12.1 \\
2 & 8.9 & 20.0 & 51.1 & 20.0 \\
3 & 50.0 & 30.0 & 15.7 & 4.3 \\
4 & 15.2 & 73.9 & 10.9 & 0.0 \\
5 & 100.0 & 0.0 & 0.0 & 0.0 \\
6 & 34.8 & 51.5 & 13.6 & 0.0 \\
7 & 17.9 & 71.4 & 8.9 & 1.8 \\
8 & 10.5 & 71.9 & 14.0 & 3.5 \\
9 & 21.9 & 48.4 & 18.7 & 10.9 \\
10 & 27.7 & 60.0 & 7.7 & 4.6 \\
11 & 19.3 & 41.9 & 30.6 & 8.1 \\
12 & 59.4 & 34.8 & 4.3 & 1.4 \\
13 & 20.0 & 34.3 & 30.0 & 15.7 \\
14 & 66.0 & 26.0 & 8.0 & 0.0 \\
\hline
\end{tabular}

Note-In relation to each maze arm, there is only one arm separated by four, but two arms separated by each of the other three values. 
maze arm were assigned a value of 2 , and so on. It should be noted that there was only one maze arm with a value of 4 (directly across the central area from the previously chosen arm), but two maze arms in each of the other three categories. With the exception of Rat 5 , subjects do not appear to have moved from maze arm to maze arm in a consistent pattern.

\section{Discussion}

The major result of this experiment is that the choices of rats were very accurate-in the same range as the choice accuracy of rats in numerous experiments in which extramaze visual cues were freely available. Thus, in agreement with conclusions based on results from other laboratories (Foreman, 1985; Whishaw \& Tomie, 1989; Zoladek \& Roberts, 1978), these results show that performance in the RAM can include above-chance choice accuracy (in fact, very high levels of choice accuracy) even when visual extramaze cues are not available.

Two explanations for this ability, other than the use of intrinsic cues by a process such as dead reckoning, are possible. First, rats could have been exhibiting response tendencies that reduced the probability of arm revisits. One rat clearly did so, as can be seen in Table 1. During the last block of trials, this rat always moved from one maze arm to an immediately adjacent arm (and although we have not shown this in the table, it always moved to an arm in the same direction). Thus, this rat never revisited an arm during the last block of trials. This rat was the exception, however. Most rats did not show clear, strong response patterning, nor were there any clear tendencies across rats. Thus, these data suggest that response patterning does not explain the high levels of choice accuracy found. However, without a more sophisticated analysis of these response distributions, it remains possible that response tendencies are involved in the choice accuracy shown in this experiment (and any other RAM experiment using a free-choice procedure).

A second possible explanation for the ability of these rats to avoid revisits is that they used intramaze cues, either those available due to imperfections in the construction of the maze or physical traces left by the rats themselves (i.e., an odor trail). Although the maze was rotated to a randomly chosen orientation prior to each trial, the physical identity of each maze arm was confounded with spatial location within each trial.

\section{EXPERIMENT 2}

Experiment 2 was designed to test the two alternatives to intrinsic cues as explanations for the choice accuracy of rats in this modified RAM. This was done by using the forced-choice procedure employed in a large number of previous RAM experiments to rule out involvement of intramaze cues and response tendencies. During each trial, the rat was first allowed to visit four randomly chosen maze arms. Then, during a brief trial interruption, the rat was removed from the maze and the maze was rotated so that each spatial location contained a different maze arm than it had during the forced choices. The rat's ability to avoid revisits to spatial locations to which it had been forced was then reinforced and measured.

\section{Method}

Subjects and Apparatus. The same 14 rats used in Experiment 1 participated in the experiment. The apparatus was the same as that used in Experiment 1.

Procedure. The experiment began immediately following the completion of Experiment 1. Prior to each trial, the maze was rotated to one of eight randomly chosen orientations. Four of the eight locations were randomly chosen to be the forced locations. These four locations were each baited with two pellets, and access to the remaining four locations was blocked using white styrofoam food containers that fit snugly into the entrance of each arm from the central arena. The rat was first placed in the central arena, as in Experiment 1 . It was allowed to choose from among the four available arms until each had been chosen. Upon its return to the central arena following its final choice, the lid was opened and the rat was removed from the maze and placed in an opaque holding cage, similar to its home cage. The experimenter then rotated the maze to one of the eight orientations, randomly chosen. He removed the blocks from the arm entrances and baited the arms in the spatial locations that had not been visited during the forced choices (and ensured that the other four spatial locations did not contain pellets). This operation took approximately $1 \mathrm{~min}$, after which the rat was returned to the central arena of the maze and allowed to choose from among the eight arms until the four baited arms had been chosen or $3 \mathrm{~min}$ had elapsed without a choice. Each rat wăs tested for 30 daily trials.

\section{Results}

Rats failed to complete a trial (because they ceased making choices for $3 \mathrm{~min}$ ) on a mean of 1.2 of the 30 triais (range $=0-6$ ). The mean number of choices required to choose the four baited maze arms during the free choices of the remaining trials is shown in the top panel of Figure 3. As always in RAM research, choice of an appropriate estimate of chance performance for comparison of these data depends on one's assumptions about the role and meaning of response tendencies. However, in order to provide some context for evaluating the overall choice accuracy of rats in this experiment, we used a Monte Carlo simulation that determined that if rats were given four forced choices and then chose randomly from among the eight arms, they should make 17.0 choices before visiting the four remaining arms. The mean empirical value (7.1) differed reliably from this estimate $[t(13)=$ $43.3, p<.001]$. The mean empirical values for the three trial blocks were not reliably different $[F(2,26)=3.21$, $p=.06]$.

To examine the control of choices by the spatial locations and the physical maze arms visited as forced choices, probabilities of visiting maze arms in each of four classes (one or more times) during the first four free choices following the trial interruption were determined. The four classes of arms were defined by whether or not the maze arm in that spatial location had been visited as a forced choice and whether or not that location had been visited as a forced choice. These probabilities are shown in the bottom panel of Figure 3. The probability of revisiting 

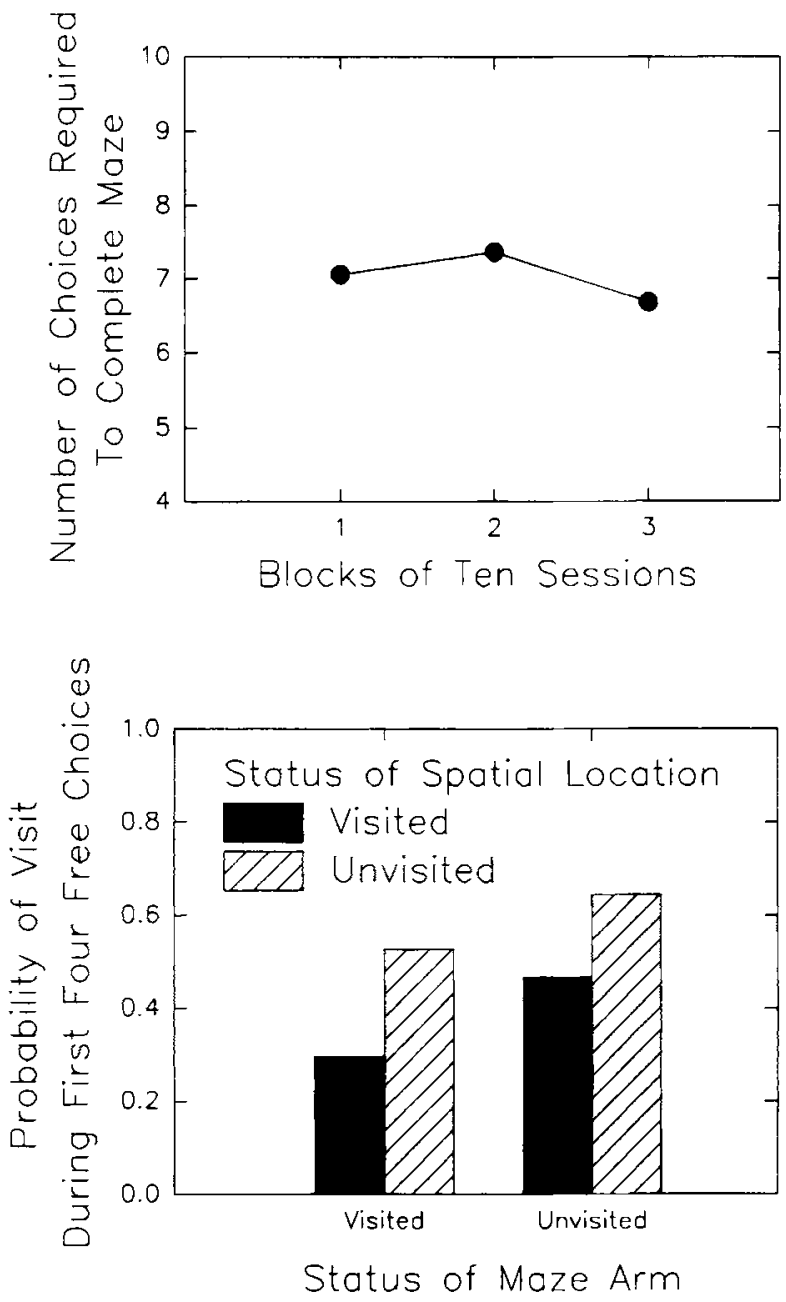

Figure 3. Choice accuracy during Experiment 2 (top panel) and probabilities of visiting maze arms during the first four free choices of Experiment 2 trials as a function of whether the physical maze arm and its spatial location had been visited during the forced choices (bottom panel).

maze arms that had been visited as forced choices was lower than the probability of visiting maze arms that had not been $[F(1,13)=38.3, p<.001]$. The probability of revisiting spatial locations that had been visited as forced choices was lower than the probability of visiting spatial locations that had not been $[F(1,13)=29.9, p<.001]$. There was no evidence that the effects of these variables interacted $[F(1,13)=3.1]$.

\section{Discussion}

These results show clearly that rats avoided both physical maze arms and spatial locations that had been visited during the forced-choice phase of an experimental trial. Although the rotation procedure rendered the identity of the maze arms to which a rat had been forced irrelevant, rats apparently avoided those particular arms by using either some subtle physical feature that differentiated among the eight maze arms or a physical trace (e.g., an odor trail) left behind during the forced choices. The use of such cues would have been reinforced during Experiment 1 , during which the maze remained in the same orientation throughout each trial.

However, the rats also avoided revisits to spatial locations that had been visited during the forced choices, regardless of whether or not the maze arm in that location had been visited. The techniques used in this experiment were designed to eliminate all extra-apparatus spatial cues. The forced-choice procedure (in particular, the fact that the set of forced locations was randomly selected on each trial) rules out response tendencies as an explanation for this ability. The rotation of the maze during the trial interruption allowed assessment of avoidance of physical maze arms independently of avoidance of particular spatial locations. Thus, discrimination of spatial locations visited during the forced choices from those not visited must have been controlled by cues generated and maintained within the rat, presumably produced by the movement of the rat as it visited locations. These cues are therefore inferred to be kinesthetic or vestibular in nature.

It should be emphasized that the rat was removed from the maze during the brief trial interruption. It would be expected that this removal and handling procedure would disrupt the rat's ability to use kinesthetic or vestibular cues to determine location upon being returned to the maze. It is possible that the exposure to the visual cues in the room during removal from the maze were somehow involved in establishing a "heading" (Margules \& Gallistel, 1988) that allowed the rat to determine its orientation after being returned to the maze. Alternatively, the rat might have maintained a heading established in the maze during the time that it was removed from the maze, placed in the holding cage, removed from the holding cage, and then returned to the maze. In any event, the use of intrinsic cues in a complex navigational problem such as the RAM is particularly striking given the trial interruption and removal from the maze used in this experiment.

\section{EXPERIMENT 3}

The results thus far indicate that intrinsic cues are sufficient to support discrimination of locations in an 8-arm maze, although the level of discrimination appears to be substantially lower than that typically found in a standard RAM. The present experiment was concerned with how intrinsic cues and visual cues interact in the control of spatial choice. It may be that control by intrinsic cues occurred in Experiments 1 and 2 only because visual cues were not available. Alternatively, it may be that intrinsic cues are usually involved in RAM performance. According to this view, control by intrinsic cues is not apparent in a standard RAM because they are confounded with extrinsic visual cues.

The apparatus used in these experiments provided an opportunity to measure control by intrinsic cues and control by visual cues separately. Explicit visual cues were 
added to each maze arm. In the standard configuration, each visual stimulus corresponded to a particular spatial location. Each trial began with three forced choices to arms with the visual cues in the standard configuration. The rat was then removed from the maze for a brief delay, during which the maze could be rotated and the stimuli could be moved. These procedures allowed control by intramaze cues, visual cues, and spatial location to be measured independently. During the first phase of the experiment, spatial locations and visual cues corresponded, as they do in a standard RAM procedure. The maze was rotated during the delay in order to dissociate any intramaze cues from other cues, but after rotation, the visual cues were returned to the standard configuration. During the second phase of the experiment, visual cues were in the standard configuration during the forced choices. However, following the delay, the visual cues were placed in nonstandard spatial locations. Thus, the spatial locations, visual cues, and intramaze cues corresponding to arms visited during the forced choices were factorially manipulated. The arms of the maze were baited on the basis of the stimuli they contained; arms containing stimuli that had been visited during the forced choices were not baited, whereas those that contained stimuli not visited during the forced choices were baited. Thus, the contingencies of reinforcement encouraged rats to avoid revisits to maze arms containing stimuli that had been visited during the forced choices.

During Phase 2 of the experiment, visual cues were moved during the delay (relative to the standard configuration) in two different ways. During some trials, the cues were rotated relative to the standard configuration, but they maintained the same spatial relations to one another. During other trials, the cues were randomly located. This manipulation corresponds to that used by Suzuki et al. (1980), who found that maintenance of the spatial relations among cues allowed them to continue controlling choices, whereas disruption of such spatial relations resulted in a lack of control by spatial cues. Part of the original purpose of the present experiment was to apply Suzuki et al.'s logic in a context that would allow better control of the stimuli (Moore, 1996).

\section{Method}

Subjects. The subjects were 12 experimentally naive male Sprague-Dawley rats obtained from the same source and maintained in the same manner as those used in Experiments 1 and 2. They were 3 months old when the experiment began. Experimental procedures occurred during the dark phase of the cycle.

Apparatus. The apparatus was the same as that used in Experiments 1 and 2 except that liners were used to add visual stimuli to the arms of the maze. Two identical sets of liners were constructed using laminated posterboard. These were constructed to be placed in the maze arms in such a way that the rats walked directly on them. The liners extended from the stem of each arm to its end. Each set of liners consisted of the following black-and-white forms: textured black, wavy 4-mm-wide stripes parallel to the axis of the maze arm (constructed from Chartpack, Inc., Pattern PT159), 1.4. $\mathrm{cm}$-wide stripes perpendicular to the axis of the maze arms, solid black, a checkerboard pattern with elements $5.4-\mathrm{cm}$ square, black dots $4 \mathrm{~cm}$ in diameter and separated by $10 \mathrm{~cm}$, a pattern of $1-\mathrm{mm}$ elements separated by $4 \mathrm{~mm}$ (Chartpack Pattern PT I33), and a pattern of irregular white shapes averaging approximately $.5 \mathrm{~cm}$ in diameter (Chartpack Pattern PT118). The eighth arm was specified by the absence of a liner (it therefore was solid white). These stimuli are listed in an order that corresponds to their spatial relations (stimuli listed consecutively were adjacent in space in the standard configuration). To ensure that the stimuli were sufficiently illuminated, an incandescent light ( $15 \mathrm{~W}$ ) was mounted in the center of the central area on the surface of the bedding material. This light rotated along with the maze.

Training. Rats were given the sucrose pellets to be used as reinforcement in their home cages for 3 days prior to training. For each of 3 consecutive days, the rats were allowed to explore the maze in groups of cagemates for 10-15 min per day. During training, one of the two sets of liners was used, and the liners were in the standard configuration. However, the maze was rotated into a randomly selected orientation (of the eight equally spaced possibilities) prior to each trial. Thus, the stimulus liners were always in the same spatial locations (the standard configuration), but the maze arms were not. This was accomplished by removing liners from maze arms and placing them in the arm occupying the appropriate spatial location. Food pellets were scattered in the central arena and down the length of each maze arm, and two pellets were placed in each food cup. Beginning the following day, rats were placed individually in the maze, with two pellets placed in each food cup and with several scattered along each maze arm. The first trial in which a rat consumed the pellets from at least one food cup was considered the last trial of training. Phase 1 began the following day.

Phase 1. Prior to each daily trial, two pellets were placed in each food cup. As in training, the liners were in the standard configuration throughout Phase 1 . However, the maze arms were rotated into a randomly selected orientation. Each trial began with three forced choices, using the same procedure that had been used during Experiment 2 (except that three rather than four forced choices occurred). The rat was removed from the maze for approximately $2.5 \mathrm{~min}$ and placed in a small cage while the maze was rotated to a new orientation, randomly selected from among the eight possibilities with the constraint that it not be the same as the orientation in place during the forced choices. During this delay, the stimulus liners were moved so that they occupied the same spatial locations as they had during the forced choices, and the reinforcement pellets were moved so that there were pellets in food cups at the ends of arms in locations (and therefore with stimuli) that had not been visited during the forced choices. The rat was returned to the central arena and allowed to choose freely from among the eight maze arms until all baited arms had been visited, 20 choices had been made, or 5 min elapsed without a choice. It should be emphasized that, as shown in the top panel of Figure 4, the stimuli stayed in the standard configuration throughout Phase 1 of the experiment, but maze arms were rotated into different and unpredictable spatial locations during the forced-choice and free-choice parts of each trial. Phase I was conducted for 20 trials ( 1 trial per day).

Phase 2. Phase 2 trials were conducted identically to those of Phase 1 except for the movement of the liners during the delay. Stimuli were moved in two ways, corresponding to the manner in which stimuli were manipulated in the experiments of Suzuki et al. (1980). In the rotation condition, the spatial locations of stimuli were rotated $180^{\circ}$ relative to the standard configuration in such a way that the stimuli maintained the same spatial relations to one another but were in different spatial locations. In the transposition condition, the spatial location of each stimulus was randomly determined. with the constraint that there be one stimulus per spatial location. These manipulations are illustrated in the bottom panel of Figure 4. A randomly selected set of 6 rats received five trials in the rotation condition, followed by five trials in the transposition condition. The remaining 6 rats received the conditions in the opposite order. To ensure that odor or other cues left on the liners did not affect performance, one of the two sets of liners was used during the 
PHASE 1

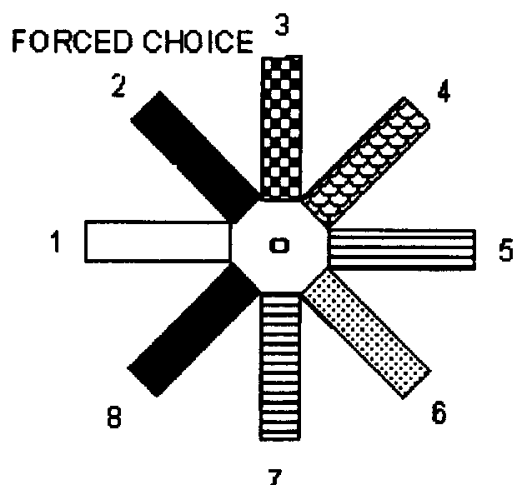

7

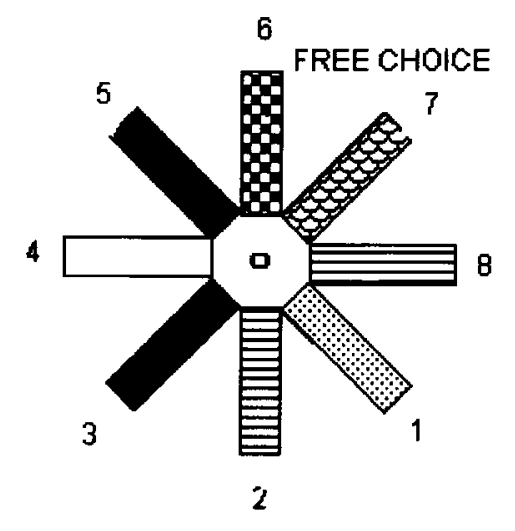

PHASE 2

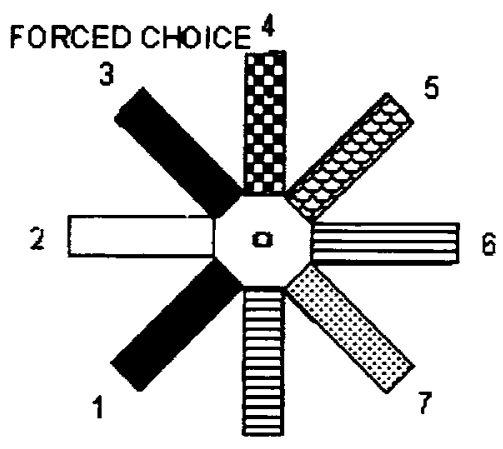

8

FREE CHOICE: ROTATION 8

2

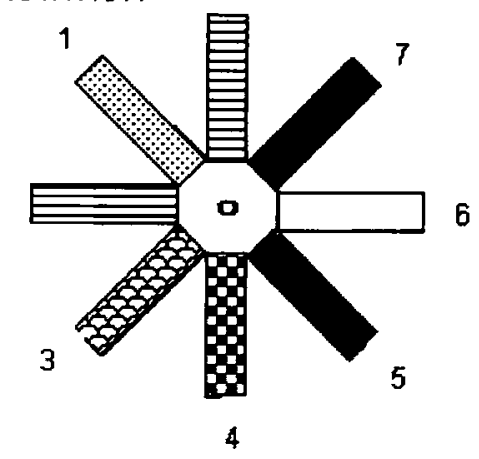

\section{FREE CHOICE:}

TRANSPOSITION

8

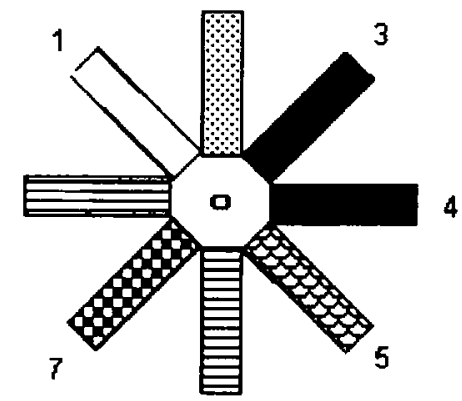

6

Figure 4. Schematic representation of the relationships among spatial location, stimulus liners, and physical maze arms during Phase 1 (top panel) and Phase 2 (bottom panel) of Experiment 3. Numbers represent the identity of physical maze arms, which were rotated into unpredictable locations during the forced and free choices of both phases. Spatial locations of the maze arms in the experiment are represented by the orientation of the maze arms in the figure. Stimulus liners were in a standard configuration during the forced choices of both phases and the free choices of Phase 1, but were either rotated or transposed during the free choices of Phase 2 . Textures representing stimulus liners do not all correspond to the actual stimulus liners used in the experiment. Drawings are not to scale. 


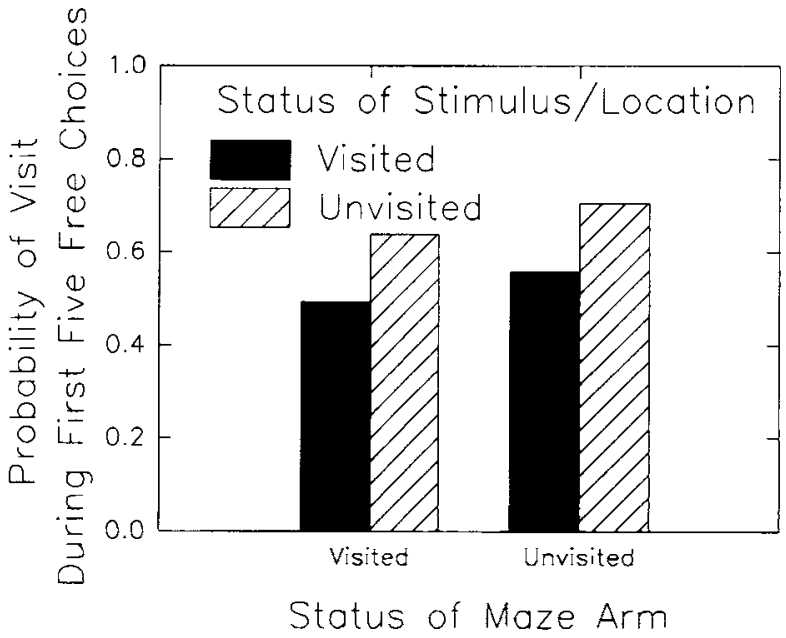

Figure 5. Probabilities of visiting maze arms during the first five free choices of trials during Phase 1 of Experiment 3 as a function of whether the physical maze arm and its spatial location (along with the corresponding stimulus) had been visited during the forced choices.

forced choices (prior to stimulus movement), and the second set was used during the free choices (after stimulus movement).

As in Phase 1, the maze was rotated during the delay as well. The fact that the maze and the stimulus liners were moved independently during the delay allowed stimulus control by three aspects of the maze arms to be dissociated: (1) whether or not the physical arm had been visited during the forced choices (an effect would indicate control by intramaze cues), (2) whether or not the stimulus in the arm had been visited during the forced choices (an effect would indicate control by the stimuli), and (3) whether or not the spatial location had been visited during the forced choices (an effect would indicate control by intrinsic cues). Following maze rotation and stimulus movement, maze arms could be classified into one of the eight categories defined by the $2 \times 2 \times 2$ combination of these factors.

\section{Results}

Training. Training was very rapid. All rats completed the training phase within 10 trials.

Phase 1. During Phase 1, there was a mean (over rats) of 1.4 trials during which rats failed to choose all five of the baited arms during the free choices (range $=0-6$ ). During the trials that were completed, a mean (over rats) of 7.75 choices was required to locate the five baited arms. Rats that were given three forced choices and then allowed to choose randomly from among the eight arms were expected to require a mean of 18.4 choices to locate the remaining five arms (this value is based on a Monte Carlo simulation). The empirical value differed reliably from this estimate of chance $[t(11)=121.5, p<.001]$.

To determine what controlled these choices, an analysis identical to that performed for Experiment 2 was conducted. The probabilities of visiting four classes of maze arms (one or more times) during the first five free choices following the trial interruption were determined. The four classes of arms were defined by whether or not that spatial location (and therefore the visual cue) had been visited as a forced choice and whether or not a physical maze arm (which now contained a different visual cue) had been visited as a forced choice. These probabilities are shown in Figure 5. The probability of revisiting maze arms that had been visited as a forced choice was slightly but reliably lower than the probability of visiting maze arms that had not been $[F(1,11)=9.1, p<.05]$. The probability of revisiting spatial locations (and the corresponding visual stimuli) that had been visited as forced choices was lower than the probability of visiting spatial locations that had not been $[F(1,11)=19.5, p<.001]$. There was no evidence that the effects of these variables interacted $[F(1,11)=0.0]$.

There was a pronounced tendency for rats to choose arms adjacent to the last choice during the free choices. Specifically, a mean (over rats) of $84.2 \%$ of free choices (not counting the initial free choice) was to an arm adjacent to the previous choice (range over rats $=62.7 \%-$ 97.6\%).

Phase 2. There was no evidence that stimulus movement condition affected performance. During the first five free choices, rats in the rotation and transposition conditions chose a mean of 3.1 and 3.05 arms containing stimuli that had not been visited during the forced choices, respectively $[t(11)<1]$. Thus, stimulus movement conditions were combined in the following analyses.

To determine whether the stimuli or spatial location controlled choices when the two were dissociated, the probability of visiting a maze arm during the free choices was determined as a function of whether that spatial location had or had not been visited during the forced choices, whether the visual stimulus in the arm had or had not been visited during the forced choices, and whether the physical arm itself (now containing a different visual cue) had or had not been visited during the forced choices. These probabilities are shown in Figure 6. There was no evidence for an effect of location, stimulus, or physical maze arm, nor was there evidence for any interactions among the effects of these variables [all $\left.F_{\mathrm{s}}(1,11)<1\right]$.

Given that the status of the stimulus in a maze arm did not control choices, the ability of rats to avoid revisits to stimuli visited during the forced choices should not have differed from chance. Given that five of the eight $(62.5 \%)$ arms were baited following the delay, rats would be expected to have visited $(5 \times .625=) 3.125$ maze arms containing unvisited stimuli (i.e., baited) during the first five free choices (assuming no arm was visited twice during those five choices). This estimate does not differ from the actual value (3.1). As in Phase 1, rats had a tendency to visit arms adjacent to the arm chosen most recently. Specifically, a mean (over rats) of $81.0 \%$ of free choices (not counting the initial free choice) were to an arm adjacent to the previous choice (range over rats $=63.9 \%-$ $90.4 \%)$.

\section{Discussion}

In Phase 1 of this experiment, choices were controlled by previous (forced) visits to the physical maze arm and by previous visits to the stimulus and/or spatial location 

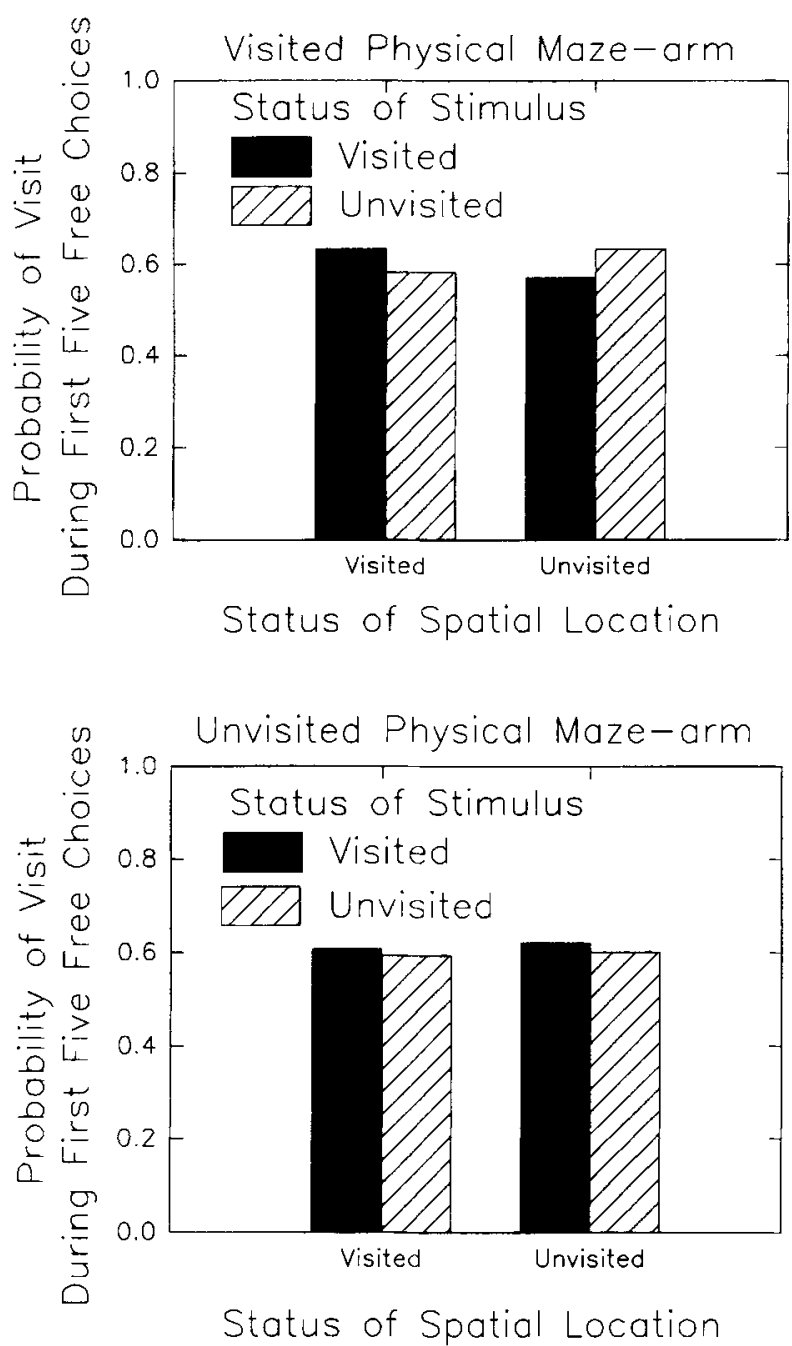

Figure 6. Probabilities of visiting maze arms during the first five free choices of trials during Phase 2 of Experiment 3 as a function of whether the physical maze arm, its spatial location, and the stimulus moved to that location had been visited during the forced choices.

in (of) a maze arm. In Phase 2 of the experiment, the stimulus in a maze arm was unconfounded with the spatial location of the arm because the stimuli were moved. Under these conditions, there was no evidence of control by the stimulus, the spatial location, or the maze arm. Thus, it appears that movement of the visual stimuli from their standard spatial locations disrupted the stimulus control that occurred when the stimuli were in their standard locations.

The difference in the results of Phases 1 and 2 can be understood if it is hypothesized that the spatial location of a maze arm and the stimuli occupying that location are represented in an integrated fashion, in such a way that moving the nominal stimulus disintegrates or distorts the effective stimulus that had been controlling behavior. Brown and Bing (1997) have provided evidence that intramaze and extramaze stimuli can been repre- sented in such an integrated fashion, producing disruption of choice performance when a RAM is rotated during a delay. Such disintegration of intramaze features from other aspects of the representation controlling behavior may have produced the apparently lower levels of choice accuracy found in Experiment 2 relative to those in Experiment 1. Likewise, disintegration of the visual stimuli from other aspects of the representation controlling behavior could have produced a loss of control in Phase 2 of the present experiment relative to the control found in Phase 1. Of course, the "other aspects of control" in the present experiment are features that specify spatial location in the absence of any extrinsic cues. By elimination, this implicates the use of intrinsic (vestibular or kinesthetic) cues. Thus, we propose that the loss of stimulus control of choices in Phase 2 (relative to Phase 1) can be explained only if such intrinsic cues were involved in the control of choices during Phase 1. This conclusion provides converging evidence for the conclusion reached on the basis of the data of Experiments 1 and 2.

Although the present experiment did allow stimulus control of choices to be detected, the magnitude of that control (as measured by choice accuracy during Phase 1) seems rather low. This is no doubt related to the pronounced tendency of the rats to choose adjacent maze arms in the present experiment. The reason for this tendency in not known, but might be attributed to the light mounted in the central arena of the maze. This light restricted movement in the central arena. It should be noted, however, that the loss of control in Phase 2 relative to Phase 1 cannot be attributed to an increase in this adjacent arm bias because the bias was not larger during Phase 2.

There was no evidence that the manner in which the stimuli were moved during the delay affected performance. This manipulation was originally intended to extend Suzuki et al.'s (1980) finding that transposition of stimuli produced a larger disruption of choice accuracy than did rotation. The present failure to replicate this finding must be interpreted with caution, however, given that there was no evidence for any control of choices by the stimuli during Phase 2.

\section{GENERAL DISCUSSION}

Two findings from these experiments support the conclusion that rats discriminated among the eight arms of the maze on the basis of intrinsic (vestibular or kinesthetic) cues. First, choices during the free choices of Experiment 2 were controlled by spatial location despite the facts that (1) no extramaze visual cues were available (due to the construction of the maze), (2) the use of response algorithms or biases was ruled out (by the forcedchoice procedure), and (3) control by intramaze cues (such as an odor trail) was accounted for. Second, when stimuli corresponding to particular spatial locations (during Phase 1 of Experiment 3) occurred in unpredictable 
spatial locations (during Phase 2 of Experiment 3), rats that had been controlled by some aspect of the stimulus/location complex came to choose randomly with respect to both stimuli and spatial locations.

Compelling behavioral evidence indicates that rats and other animals use intrinsic kinesthetic or vestibular cues to find their way back to a starting point after traveling even long and complex paths (Gallistel, 1990; Mittelstaedt \& Mittelstaedt, 1982). As noted, several previous studies suggest the seemingly more complex ability of rats (and hamsters) to use dead reckoning to discriminate between visited and unvisited arms in the RAM. With the exception of Zoladek and Roberts's (1978) study, however, these experiments have failed to rule out alternatives to dead reckoning as an explanation for choice accuracy. The present study confirms Zoladek and Roberts's preliminary result that above-chance choice accuracy can be obtained even when access to extrinsic cues is eliminated, indicating that intrinsic cues are sufficient to discriminate among at least several of the distinct locations corresponding to the arms of an 8-arm RAM.

Other recent lines of research are consistent with this conclusion. Ossenkopp and Hargreaves (1993) induced vestibular deficits using chemical labyrinthectomies. Rats with such deficits performed substantially less accurately in an 8-arm RAM than did control rats. This result shows that the vestibular system is somehow involved in RAM performance, although it is possible that the mechanism of this effect does not specifically correspond to rats' ability to discriminate among spatial locations. There is also growing physiological evidence that spatial orientation ("heading") is directly coded in several areas of the nervous system (see, e.g., Lavoie \& Mizumori, 1994; McNaughton, Chen, \& Markus, 1991; Taube, Muller, \& Ranck, 1990). Representation of heading is a prerequisite for the use of dead reckoning. These findings, together with the present ones, converge on the conclusion that intrinsic cues can allow rats to discriminate the location of previously visited maze arms from those not previously visited in the RAM.

The results of Experiment 3 indicate that the intrinsic cues guiding performance in the earlier experiments and extrinsic visual cues do not operate independently, but are instead integrated in a manner that produces disruption of stimulus control when the two types of cues are in disagreement. A theory of how vestibular and visual information might be integrated into a spatial representation was proposed by McNaughton et al. (1991). An important feature of their model is the use of visual landmarks to calibrate the dead-reckoned estimate of location. This seems to be critical because of the increasingly poor estimate of location that would be expected due to cumulative error in the dead reckoning system. In this context, it is of interest to emphasize that rats in the present RAM had no access to visual cues throughout each trial. Thus, it may be that the relatively low (albeit above- chance) levels of choice accuracy obtained in Experiment 2 and in Phase 1 of Experiment 3 were due to imprecise estimates of locations provided by the dead reckoning mechanism in the absence of extrinsic cues. The interaction between intrinsic and extrinsic information in the discrimination of spatial locations is worthy of additional experimental attention.

\section{REFERENCES}

Brown, M. F. (1992). Does a cognitive map guide choices in the radialarm maze? Journal of Experimental Psychology: Animal Behavior Processes, 18, 56-66.

Brown, M. F. (1993). Sequential and simultaneous choice processes in the radial-arm maze. In T. Zentall (Ed.), Animal cognition: $A$ tribute to Donald A. Riley (pp. 153-173). Hillsdale, NJ: Erlbaum.

Brown, M. F., \& Bing, M. N. (1997). In the dark: Spatial choice when access to spatial cues is restricted. Animal Learning \& Behavior, 25, 21-30.

Brown, M. F., Rish, P. A., VonCulin, J. E., \& Edberg, J. A. (1993). Spatial guidance of choice behavior in the radial-arm maze. Journal of Experimental Psychology: Animal Behavior Processes, 19 195-214.

DALE, R. H. I., \& INNIS, N. K. (1986). Interactions between response stereotypy and memory strategies on the eight-arm radial maze. Behavioural Brain Research, 19, 17-25.

Eckerman, D. A. (1980). Monte Carlo estimation of chance performance for the radial arm maze. Bulletin of the Psychonomic Society, 15, 93-95.

EtiENNE, A. S. (1992). Navigation of a small mammal by dead reckoning and local cues. Current Directions in Psychological Science, 1 , 48-52.

Etienne, A. S., Sitbon, S., Dahn-Hurni, C., \& Maurer, R. (1994). Golden hamsters on the eight-arm maze in light and darkness: The role of dead reckoning. Quarterly Journal of Experimental Psychology, 47B, 401-425.

FOREMAN, N. (1985). Algorithmic responding on the radial maze in rats does not always imply absence of spatial encoding. Quarterly Journal of Experimental Psychology, 37B, 333-358.

Gallistel, C. R. (1990). The organization of learning. Cambridge, MA: MIT Press.

Goodale, M. A., \& Dale, R. H. I. (1981). Radial-maze performance in the rat following lesions of posterior neocortex. Behavioural Brain Research, 3, 273-288.

LAvoie, A. M., \& Mizumori, S. J. Y. (1994). Spatial, movement- and reward-sensitive discharge by medial ventral striatum neurons of rats. Brain Research, 638, 157-168.

Margules, J., \& Gallistel, C. R. (1988). Heading in the rat: Determination by environmental shape. Animal Learning \& Behavior, 16, 404-410.

Mazmanian, D. S., \& Roberts, W. A. (1983). Spatial memory in rats under restricted viewing conditions. Learning \& Motivation, 14, 123 139.

McNaughton, B. L., Chen, L. L., \& Markus, E. J. (1991). "Dead reckoning," landmark learning, and the sense of direction: A neurophysiological and computational hypothesis. Journal of Cognitive Neuroscience, 3, 190-202.

Mittelstaedt, H., \& MitTelstaedt, M. L. (1982). Homing by path integration. In H. Papi \& G. Wallraff (Eds.), Avian navigation (pp. 290-297). Berlin: Springer-Verlag.

MOORE, J. A. (1996). Guidance of choices by spatial relations among visual cues: A re-evaluation of a critical study. Unpublished Master's thesis, Villanova University.

OLTON, D. S., \& CoLLISON, C. (1979). Intramaze cues and "odor trails" fail to direct choice behavior on an elevated maze. Animal Learning \& Behavior, 7, 221-223.

Olton, D. S., \& Samuelson, R. J. (1976). Remembrance of places passed: Spatial memory in rats. Journal of Experimental Psychology: Animal Behavior Processes, 2, 97-116. 
OSSENKOPP, K., \& HaRGREAVES, E. L. (1993). Spatial learning in an enclosed eight-arm radial maze in rats with sodium arsanilate-induced labyrinthectomies. Behavioral \& Neural Biology, 59, 253-257.

PoTEGAL, M. (1982). Vestibular and neostriatal contributions to spatial orientation. In M. Potegal (Ed.), Spatial abilities: Development and physiological foundations (pp. 361-387). New York: Academic Press.

Suzuki, S., Augerinos, G., \& Black, A. H. (1980). Stimulus control of spatial behavior on the eight-arm maze in rats. Learning \& Motivation, 11, 1-18.

TAUBe, J. S., Muller, R. U., \& RanCK, J. B., JR. (1990). Head-direction cells recorded from the postsubiculum in freely moving rats: I. De- scription and quantitative analysis. Journal of Neuroscience, 10, 420435.

Whishaw, I. Q., \& Tomie, J. (1989). Food-pellet size modifies the hoarding behavior of foraging rats. Psychobiology, 17, 93-101.

ZOLADEK, L., \& ROBERTS, W. A. (1978). The sensory basis of spatial memory in the rat. Animal Learning \& Behavior, 6, 77-81.

(Manuscript received September 29, 1996; revision accepted for publication February $21,1997$. 